\title{
On the Partial Sums of the q-Generalized Dini Function
}

\author{
Alaa H. El-Qadeem $\mathbb{D}^{1},{ }^{1}$ Mohamed A. Mamon $\mathbb{D}^{2},{ }^{2}$ and Ibrahim S. Elshazly $\mathbb{D}^{3}$ \\ ${ }^{1}$ Department of Mathematics, Faculty of Science, Zagazig University, Zagazig 44519, Egypt \\ ${ }^{2}$ Department of Mathematics, Faculty of Science, Tanta University, Tanta 31527, Egypt \\ ${ }^{3}$ Department of Basic Sciences, Common First Year, King Saud University, Alriyad 11451, Saudi Arabia \\ Correspondence should be addressed to Alaa H. El-Qadeem; ahhassan@science.zu.edu.eg
}

Received 29 December 2021; Accepted 29 January 2022; Published 21 February 2022

Academic Editor: V. Ravichandran

Copyright (c) 2022 Alaa H. El-Qadeem et al. This is an open access article distributed under the Creative Commons Attribution License, which permits unrestricted use, distribution, and reproduction in any medium, provided the original work is properly cited.

Our objective in this paper is to introduce a q-analog of the generalized Dini function. Also, we investigate the lower bound for the ratio of the q-generalized Dini function to its sequences of partial sums.

\section{Introduction and Basic Concepts}

Let $\mathscr{A}$ denote the class of functions $f$ that have the following Maclaurin's form,

$$
f(z)=z+\sum_{n=2}^{\infty} a_{n} z^{n}
$$

which is analytic and univalent in the open unit disc $\mathscr{U}=$ $\{z:|z|<1\}$ and satisfies the normalization conditions $f(0)=f^{\prime}(0)-1=0$.

Special functions play an inspired role in applied mathematics and physics. The widespread use of these functions has attracted many researchers to work in many directions. Lately, many authors studied the geometric properties of some special functions such as starlikness, univalence, and convexity, see [1-6]. There are several results related to partial sums of analytic univalent functions that were developed by the authors in [7-9]. Specifically, the authors in [10] investigated the partial sums of the generalized Bessel function, and then, a lot of authors followed them in studying the same problem for different special functions such as Bessel [11, 12], Struve [13], Lommel [14], Wright [15], and Mittag-Leffler [16], see also [17].

Our aim in this study is to develop a q-analog of the generalized Dini function, which is inspired by early studies on analytic and special functions. We also provide lower bounds for the ratio of $q$-generalized Dini function to its sequences of partial sums, for $m \in \mathbb{N}=\{1,2,3, \ldots\}$, $\left(\psi_{v, b, c}^{a}(z ; q)\right)_{m}=z+\sum_{n=1}^{m} \zeta_{n} z^{n+1}$. We will investigate the following:

$$
\mathfrak{R}\left\{\frac{\psi_{\nu, b, c}^{a}(z ; q)}{\left(\psi_{\nu, b, c}^{a}(z ; q)\right)_{m}}\right\}, \mathfrak{R}\left\{\frac{\left(\psi_{\nu, b, c}^{a}(z ; q)\right)_{m}}{\psi_{\nu, b, c}^{a}(z ; q)}\right\}, \mathfrak{R}\left\{\frac{\left(\psi_{\nu, b, c}^{a}(z ; q)\right)^{\prime}}{\left(\psi_{\nu, b, c}^{a}(z ; q)\right)_{m}^{\prime}}\right\} \text {, and } \mathfrak{R}\left\{\frac{\left(\psi_{\nu, b, c}^{a}(z ; q)\right)_{m}^{\prime}}{\left(\psi_{\nu, b, c}^{a}(z ; q)\right)^{\prime}}\right\} \text {. }
$$

To introduce the main results, we would like to recall some fundamentals and concepts related to geometric function theory and the definition of q-generalized Bessel function. At first, let us consider the following second-order linear homogenous differential equation (for more details, see [18-20]): 


$$
\begin{array}{r}
z^{2} w^{\prime \prime}(z)+b z w^{\prime}(z)+\left[c z^{2}-v^{2}+(1-b) v\right] w(z)=0 \\
(b, c, v \in \mathbb{C})
\end{array}
$$

The function $w_{v, b, c}(z)$ is known as the generalized Bessel function of the first kind of order $v$, which is a particular solution of equation (3). The function $w_{v, b, c}(z)$ has the following series representation:

$$
w_{\nu, b, c}(z)=\sum_{n=0}^{\infty} \frac{(-c)^{n}}{n ! \Gamma(\nu+n+((b+1) / 2))}\left(\frac{z}{2}\right)^{2 n+\nu},
$$

where $\Gamma$ stands for the Gamma function.

Now, let $q \in(0,1)$ and $a \in \mathbb{C}$; the $q$-shifted factorial is defined by

$$
(a ; q)_{n}= \begin{cases}1, & n=0, \\ \prod_{k=0}^{n-1}\left(1-a q^{k}\right), & n \in \mathbb{N} .\end{cases}
$$

The limit of $(a ; q)_{n}$ as $n$ tends to infinity exists and is denoted by $(a ; q)_{\infty}$ :

$$
(a ; q)_{\infty}=\prod_{k=0}^{\infty}\left(1-a q^{k}\right) .
$$

The multiple $q$-shifted factorial for complex numbers $a_{1}, a_{2}, \ldots, a_{r}$ is defined by

$$
\left(a_{1}, a_{2}, \ldots, a_{r} ; q\right)_{n}=\prod_{j=1}^{r}\left(a_{j} ; q\right)_{n} .
$$
be

If $a q^{\alpha} \neq q^{-n}$, for all $n \in \mathbb{N}_{0}:=\mathbb{N} \cup\{0\}$, we define $(a ; q)_{\alpha}$ to

$$
(a ; q)_{\alpha}=\frac{(a ; q)_{\infty}}{\left(a q^{\alpha} ; q\right)_{\infty}}
$$

Definition 1. Let $b, v \in \mathbb{R}$ and $c \in \mathbb{C}$; the $q$-generalized Bessel function is defined by

$$
\begin{aligned}
\omega_{v, b, c}(z ; q) & =\frac{\left(q^{k} ; q\right)_{\infty}}{(q ; q)_{\infty}} \sum_{n=0}^{\infty} \frac{(-c)^{n}(1-q)^{((b-1) / 2)}}{(q ; q)_{n}\left(q^{k} ; q\right)_{n}}\left(\frac{z}{2}\right)^{2 n+v} \\
& =\frac{1}{(q ; q)_{k-1}} \sum_{n=0}^{\infty} \frac{(-c)^{n}(1-q)^{((b-1) / 2)}}{\left(q, q^{k} ; q\right)_{n}}\left(\frac{z}{2}\right)^{2 n+v}
\end{aligned}
$$

where $k=v+b+1 / 2 \neq 0,-1,-2, \ldots, z \in \mathcal{U}$ and $q \in(0,1)$.

Now, we introduce the $q$-generalized Dini function $\phi_{v, b, c}^{a}(z ; q)$ in terms of $\omega_{v, b, c}(z ; q)$.

Definition 2. Let $a \in \mathbb{R}^{+}$; the $q$-generalized Dini function $\phi_{\nu, b, c}^{a}(z ; q)$ is defined by

$$
\begin{array}{r}
\phi_{\nu, b, c}^{a}(z ; q)=(a-v) \omega_{v, b, c}(\sqrt{z} ; q)+\sqrt{z} \omega_{v, b, c}^{\prime}(z ; q), \\
=\frac{1}{(q ; q)_{k-1}} \sum_{n=0}^{\infty} \frac{(-c)^{n}(1-q)^{((b-1) / 2)}(a+2 n)}{\left(q, q^{k} ; q\right)_{n}}\left(\frac{z}{4}\right)^{(n+(v / 2))} .
\end{array}
$$

Remark 1. By specializing the value of $a, b, c$, and $q$, we see that

(1) $\lim _{q \longrightarrow 1^{-}} \omega_{v, b, c}((1-q) z ; q)=\omega_{v, b, c}(z)$ is the generalized Bessel function of first kind introduced by Orhan and Yagmur [10]

(2) By putting $b=c=1$, then $\omega_{v, 1,1}(z ; q)=J_{v}^{(1)}(z ; q)$ is the first kind of q-Bessel function given by Annaby and Mansour [21]. Also, $\lim _{q \longrightarrow 1^{-}} \omega_{v, 1,1}((1-q) z ; q)=J_{v}(z)$ is the familiar Bessel function defined by Baricz [18].

(3) By putting $b=-c=1$, then $\omega_{v, 1,-1}(z ; q)=I_{v}^{(1)}(z ; q)$ is the first kind of modified q-Bessel function given by Annaby and Mansour [21]. Also, $\lim _{q \longrightarrow 1^{-}} \omega_{v, 1,-1}((1-q) z ; q)=I_{v}(z)$ is the modified Bessel function defined by Baricz [18].

(4) $\lim _{q \longrightarrow 1^{-}} \phi_{v, b, c}^{a}\left((1-q)^{2} z ; q\right)=D_{v, a, b, c}(z)$ is the generalized Dini function investigated by Deniz and Gören [3]. Also, by putting $b=c=1$ in the last expression, we get $d_{v, a}(z)$ introduced by Aktaş and Orhan [22]. In addition, by putting $a=1$, we obtain the Dini function $d_{v}(z)$ which is introduced by Baricz et al. [2].

Because the function $\phi_{v, b, c}^{a}(z ; q)$ defined by (10) do not belong to the class $\mathscr{A}$, we consider the following normalized form of the $q$-generalized Dini function, $\psi_{\nu, b, c}^{a}(z ; q): \mathcal{U} \longrightarrow \mathbb{C}$, as

$$
\begin{aligned}
\psi_{\nu, b, c}^{a}(z ; q) & =\frac{2^{v}(q ; q)_{k-1}}{a(1-q)^{b-1 / 2}} z^{1-(v / 2)} \phi_{\nu, b, c}^{a}(z ; q) \\
& =z+\sum_{n=1}^{\infty} \zeta_{n} z^{n+1},
\end{aligned}
$$

where

$$
\zeta_{n}=\frac{(-c)^{n}(a+2 n)}{4^{n} a\left(q, q^{k} ; q\right)_{n}} .
$$

Definition 3 (subordination principle, see [23-25]). An analytic function $f$ is said to be subordinate to another analytic function $g$, written as $f(z)<g(z)(z \in \mathcal{U})$, if there exists a Schwarz function $\omega$, which is analytic in $\mathcal{U}$ with $\omega(0)=0$ and $|\omega(z)|<1 \quad(z \in \mathcal{U})$, such that $f(z)=$ $g(w(z))$. In particular, if the function $g$ is univalent in $\mathcal{U}$, then we have the following equivalence:

$$
f(z) \prec g(z) \Leftrightarrow f(0)=g(0) \text { and } f(\mathcal{U}) \subset g(\mathscr{U}) .
$$

Remark 2. It is observed that the function $\Omega(z)=1+z / 1-z$ maps $\mathcal{U}$ conformally into the right half plane such that $\mathfrak{R}\{\Omega(z)\}>0$. That function plays a great roll in proving our main results.

Here, we would like to mention the following inequalities,

$$
(q ; q)_{n} \geq(1-q)^{n}
$$


and

$$
\left(q^{\alpha} ; q\right)_{n} \geq\left(1-q^{\alpha}\right)^{n},
$$

Lemma 1. Let us consider $a \in \mathbb{R}^{+}, b, v \in \mathbb{R}, c \in \mathbb{C}(|c|<4)$, and $k=v+(b+1 / 2)$; the function $\psi_{\nu, b, c}^{a}(z ; q)$, referred by (11), satisfies the following inequalities, for all $z \in \mathscr{U}$ :

are valid for $q \in(0,1), \alpha \in \mathbb{R}$, and $n \in \mathbb{N}$.

$$
\begin{gathered}
\left|\psi_{\nu, b, c}^{a}(z ; q)\right| \leq 1+\frac{4|c|(a+2)(1-q)\left(1-q^{k}\right)-a|c|^{2}}{a\left[4(1-q)\left(1-q^{k}\right)-|c|\right]^{2}}, \\
\left|\left(\psi_{v, b, c}^{a}(z ; q)\right)^{\prime}\right| \leq 1+\frac{32|c|(a+2)(1-q)^{2}\left(1-q^{k}\right)^{2}-4|c|^{2}(3 a+2)(1-q)\left(1-q^{k}\right)+a|c|^{3}}{a\left[4(1-q)\left(1-q^{k}\right)-|c|\right]^{3}} .
\end{gathered}
$$

Proof. By taking into consideration inequalities (14) and (15), we obtain

$$
\begin{aligned}
\left|\psi_{v, b, c}^{a}(z ; q)\right|= & \left|z+\sum_{n=1}^{\infty} \frac{(-c)^{n}(a+2 n)}{4^{n} a\left(q, q^{k} ; q\right)_{n}} z^{n+1}\right| \leq 1+\sum_{n=1}^{\infty} \frac{|c|^{n}(a+2 n)}{a 4^{n}(q ; q)_{n}\left(q^{k} ; q\right)_{n}} \\
\leq & 1+\sum_{n=1}^{\infty} \frac{|c|^{n}(a+2 n)}{a 4^{n}(1-q)^{n}\left(1-q^{k}\right)^{n}} \\
& =1+\frac{|c|}{4(1-q)\left(1-q^{k}\right)} \sum_{n=1}^{\infty}\left(\frac{|c|}{4(1-q)\left(1-q^{k}\right)}\right)^{n-1}+\frac{|c|}{2 a(1-q)\left(1-q^{k}\right)} \sum_{n=1}^{\infty} n\left(\frac{|c|}{4(1-q)\left(1-q^{k}\right)}\right)^{n-1} \\
& 1+\frac{4|c|(a+2)(1-q)\left(1-q^{k}\right)-a|c|^{2}}{a\left[4(1-q)\left(1-q^{k}\right)-|c|\right]^{2}}
\end{aligned}
$$

and

$$
\begin{aligned}
\left|\left(\psi_{v, b, c}^{a}(z ; q)\right)^{\prime}\right|= & \left|1+\sum_{n=1}^{\infty} \frac{(-c)^{n}(n+1)(a+2 n)}{a 4^{n}\left(q, q^{k} ; q\right)_{n}} z^{n}\right| \leq 1+\sum_{n=1}^{\infty} \frac{|c|^{n}\left(2 n^{2}+(a+2) n+a\right)}{a 4^{n}(q ; q)_{n}\left(q^{k} ; q\right)_{n}} \\
\leq & 1+\sum_{n=1}^{\infty} \frac{\left(2 n^{2}+(a+2) n+a\right)}{a}\left(\frac{|c|}{4(1-q)\left(1-q^{k}\right)}\right)^{n} \\
= & 1+\frac{|c|}{4(1-q)\left(1-q^{k}\right)} \sum_{n=1}^{\infty}\left(\frac{|c|}{4(1-q)\left(1-q^{k}\right)}\right)^{n-1}+\frac{|c|(a+2)}{4 a(1-q)\left(1-q^{k}\right)} \sum_{n=1}^{\infty} n\left(\frac{|c|}{4(1-q)\left(1-q^{k}\right)}\right)^{n-1} \\
& +\frac{|c|}{2 a(1-q)\left(1-q^{k}\right)} \sum_{n=1}^{\infty} n^{2}\left(\frac{|c|}{4(1-q)\left(1-q^{k}\right)}\right)^{n-1} \\
= & 1+\frac{32|c|(a+2)(1-q)^{2}\left(1-q^{k}\right)^{2}-4|c|^{2}(3 a+2)(1-q)\left(1-q^{k}\right)}{a\left[4(1-q)\left(1-q^{k}\right)-|c| \mid\right]^{3}}
\end{aligned}
$$


Thus, we complete the proof.

\section{Main Results}

Theorem 1. Let us consider $a \in \mathbb{R}^{+}, \quad b, v \in \mathbb{R}$, and $c \in \mathbb{C}(|c|<4), k=v+b+1 / 2$, and the function $\psi_{v, b, c}^{a}(z ; q)$ be defined by (11) and its partial sum $\left(\psi_{v, b, c}^{a}(z ; q)\right)_{m}$ $=z+\sum_{n=1}^{m} \zeta_{n} z^{n+1}$. If the inequality,

$$
16 a(1-q)^{2}\left(1-q^{k}\right)^{2}+2 a|c|^{2}>4(3 a+2)|c|(1-q)\left(1-q^{k}\right),
$$

is valid, then

$$
\mathfrak{R}\left\{\frac{\psi_{v, b, c}^{a}(z ; q)}{\left(\psi_{v, b, c}^{a}(z ; q)\right)_{m}}\right\}>\beta_{1}
$$

and

$$
\mathfrak{R}\left\{\frac{\left(\psi_{v, b, c}^{a}(z ; q)\right)_{m}}{\psi_{v, b, c}^{a}(z ; q)}\right\}>\beta_{2}
$$

holds true, for all $z \in \mathcal{U}$, where

$$
\begin{aligned}
& \beta_{1}=\frac{16 a(1-q)^{2}\left(1-q^{k}\right)^{2}+4|c|(2-a)(1-q)\left(1-q^{k}\right)+2 a|c|^{2}}{a\left[4(1-q)\left(1-q^{k}\right)-|c|\right]^{2}}, \\
& \beta_{2}=\frac{a\left[4(1-q)\left(1-q^{k}\right)-|c|\right]^{2}}{16 a(1-q)^{2}\left(1-q^{k}\right)^{2}+4|c|(2-a)(1-q)\left(1-q^{k}\right)} .
\end{aligned}
$$

Proof. From the steps of proving Lemma 1, we observe

$$
1+\sum_{n=1}^{\infty}\left|\zeta_{n}\right| \leq 1+\frac{4|c|(a+2)(1-q)\left(1-q^{k}\right)-a|c|^{2}}{a\left[4(1-q)\left(1-q^{k}\right)-|c|\right]^{2}},
$$

which is equivalent to

$$
\mu \sum_{n=1}^{\infty}\left|\zeta_{n}\right| \leq 1
$$

where

$\mu=a\left[4(1-q)\left(1-q^{k}\right)-|c|\right]^{2} / 4|c|(a+2)(1-q)\left(1-q^{k}\right)$ $-a|c|^{2}$.

Now, let us consider

$$
\begin{aligned}
& \frac{1+\omega(z)}{1-\omega(z)}=\mu\left[\frac{\psi_{\nu, b, c}^{a}(z ; q)}{\left(\psi_{\nu, b, c}^{a}(z ; q)\right)_{m}}-\beta_{1}\right] \\
= & \frac{1+\sum_{n=1}^{m} \zeta_{n} z^{n}+\mu \sum_{n=m+1}^{\infty} \zeta_{n} z^{n}}{1+\sum_{n=1}^{m} \zeta_{n} z^{n}} .
\end{aligned}
$$

Therefore,

$$
\omega(z)=\frac{\mu \sum_{n=m+1}^{\infty} \zeta_{n} z^{n}}{2+2 \sum_{n=1}^{m} \zeta_{n} z^{n}+\mu \sum_{n=m+1}^{\infty} \zeta_{n} z^{n}}
$$

and

$$
|\omega(z)| \leq \frac{\mu \sum_{n=m+1}^{\infty}\left|\zeta_{n}\right|}{2-2 \sum_{n=1}^{m}\left|\zeta_{n}\right|-\mu \sum_{n=m+1}^{\infty}\left|\zeta_{n}\right|}
$$

Inequality (20) holds true if $|\omega(z)| \leq 1$ according to the definition of subordination. Then, the upcoming inequality,

$$
\sum_{n=1}^{m}\left|\zeta_{n}\right|+\mu \sum_{n=m+1}^{\infty}\left|\zeta_{n}\right| \leq 1
$$

implies that $|\omega(z)| \leq 1$. It suffices to show that the left-hand side of (28) is bounded above by

$$
\mu \sum_{n=1}^{\infty}\left|\zeta_{n}\right|
$$

which is equivalent to

$$
(\mu-1) \sum_{n=1}^{m}\left|\zeta_{n}\right| \geq 0 .
$$

On the contrary, to prove inequality (21), we consider

$$
\begin{aligned}
\frac{1+p(z)}{1-p(z)} & =(\mu+1)\left[\frac{\left(\psi_{v, b, c}^{a}(z ; q)\right)_{m}}{\psi_{\nu, b, c}^{a}(z ; q)}-\beta_{2}\right], \\
& =\frac{1+\sum_{n=1}^{m} \zeta_{n} z^{n}-\mu \sum_{n=m+1}^{\infty} \zeta_{n} z^{n}}{1+\sum_{n=1}^{m} \zeta_{n} z^{n}} .
\end{aligned}
$$

Therefore,

$$
p(z)=\frac{-(1+\mu) \sum_{n=m+1}^{\infty} \zeta_{n} z^{n}}{2+2 \sum_{n=1}^{m} \zeta_{n} z^{n}+(1-\mu) \sum_{n=m+1}^{\infty} \zeta_{n} z^{n}}
$$

and

$$
|p(z)| \leq \frac{(1+\mu) \sum_{n=m+1}^{\infty}\left|\zeta_{n}\right|}{2-2 \sum_{n=1}^{m}\left|\zeta_{n}\right|-(\mu-1) \sum_{n=m+1}^{\infty}\left|\zeta_{n}\right|} .
$$

Inequality (21) holds true if $|p(z)| \leq 1$ according to the definition of subordination. Then, the upcoming inequality,

$$
\sum_{n=1}^{m}\left|\zeta_{n}\right|+\mu \sum_{n=m+1}^{\infty}\left|\zeta_{n}\right| \leq 1
$$

implies that $|p(z)| \leq 1$. Since the left-hand side of (34) is bounded above $\mu \sum_{n=1}^{\infty}\left|\zeta_{n}\right|$, thus, we complete the proof. 
Theorem 2. Let us consider $a \in \mathbb{R}^{+}, b, v \in \mathbb{R}, c \in \mathbb{C}(|c|<4)$, and $k=v+b+1 / 2$, the function $\psi_{\nu, b, c}^{a}(z ; q): \mathcal{U} \longrightarrow \mathbb{C}$, be defined by (11), and its partial sum be $\left(\psi_{\nu, b, c}^{a}(z ; q)\right)_{m}$ $=z+\sum_{n=1}^{m} \zeta_{n} z^{n+1}$. If the inequalities,

$$
\left.\begin{array}{r}
32(a+2)|c|(1-q)^{2}\left(1-q^{k}\right)^{2}+a|c|^{3}>4(3 a+2)|c|^{2}(1-q)\left(1-q^{k}\right), \\
32 a(1-q)^{3}\left(1-q^{k}\right)^{3}+4(3 a+1)|c|^{2}(1-q)\left(1-q^{k}\right)>8(5 a+4)|c|(1-q)^{2}\left(1-q^{k}\right)^{2}+a|c|^{3},
\end{array}\right\}
$$

are valid, then

$$
\Re\left\{\frac{\left(\psi_{\nu, b, c}^{a}(z ; q)\right)^{\prime}}{\left(\left(\psi_{\nu, b, c}^{a}(z ; q)\right)_{m}\right)^{\prime}}\right\}>\gamma_{1}
$$

holds true, for all $z \in \mathcal{U}$, where

and

$$
\gamma_{1}=\frac{64 a(1-q)^{3}\left(1-q^{k}\right)^{3}-16|c|(5 a+4)(1-q)^{2}\left(1-q^{k}\right)^{2}+5(3 a+1)|c|^{2}(1-q)\left(1-q^{k}\right)-2 a|c|^{3}}{a\left[4(1-q)\left(1-q^{k}\right)-|c|\right]^{3}}
$$

and

$$
\gamma_{2}=\frac{a\left[4(1-q)\left(1-q^{k}\right)-|c|\right]^{3}}{64 a(1-q)^{3}\left(1-q^{k}\right)^{3}+16|c|(4-a)(1-q)^{2}\left(1-q^{k}\right)^{2}-8|c|(1-q)\left(1-q^{k}\right)} .
$$

Proof. From Lemma 1, we observe that

$$
1+\sum_{n=1}^{\infty}\left|\eta_{n}\right| \leq 1+\frac{32|c|(a+2)(1-q)^{2}\left(1-q^{k}\right)^{2}-4|c|^{2}(3 a+2)(1-q)\left(1-q^{k}\right)+a|c|^{3}}{a\left[4(1-q)\left(1-q^{k}\right)-|c|\right]^{3}},
$$

which is equivalent to where $\eta_{n}=(-c)^{n}(n+1)(a+2 n) / 4^{n} a\left(q, q^{k} ; q\right)_{n}$ and

$$
\lambda \sum_{n=1}^{\infty}\left|\eta_{n}\right| \leq 1
$$

$$
\lambda=\frac{a\left[4(1-q)\left(1-q^{k}\right)-|c|\right]^{3}}{32|c|(a+2)(1-q)^{2}\left(1-q^{k}\right)^{2}-4|c|^{2}(3 a+2)(1-q)\left(1-q^{k}\right)+a|c|^{3}} .
$$

Now, let us consider 


$$
\begin{aligned}
\frac{1+r(z)}{1-r(z)} & =\lambda\left[\frac{\left(\psi_{\nu, b, c}^{a}(z ; q)\right)^{\prime}}{\left(\left(\psi_{\nu, b, c}^{a}(z ; q)\right)_{m}\right)^{\prime}}-\gamma_{1}\right] \\
& =\frac{1+\sum_{n=1}^{m} \eta_{n} z^{n}+\lambda \sum_{n=m+1}^{\infty} \eta_{n} z^{n}}{1+\sum_{n=1}^{m} \eta_{n} z^{n}} .
\end{aligned}
$$

Therefore,

$$
r(z)=\frac{\lambda \sum_{n=m+1}^{\infty} \eta_{n} z^{n}}{2+2 \sum_{n=1}^{m} \eta_{n} z^{n}+\lambda \sum_{n=m+1}^{\infty} \eta_{n} z^{n}}
$$

and

$$
|r(z)| \leq \frac{\lambda \sum_{n=m+1}^{\infty}\left|\eta_{n}\right|}{2-2 \sum_{n=1}^{m}\left|\eta_{n}\right|-\lambda \sum_{n=m+1}^{\infty}\left|\eta_{n}\right|} .
$$

Inequality (36) holds true if $|r(z)| \leq 1$ according to subordination principle. Then, the upcoming inequality,

$$
\sum_{n=1}^{m}\left|\eta_{n}\right|+\lambda \sum_{n=m+1}^{\infty}\left|\eta_{n}\right| \leq 1
$$

implies that $|r(z)| \leq 1$. It suffices to show that the left-hand side of (47) is bounded above by

$$
\lambda \sum_{n=1}^{\infty}\left|\eta_{n}\right|
$$

which is equivalent to

$$
(\lambda-1) \sum_{n=1}^{m}\left|\eta_{n}\right| \geq 0
$$

On the contrary, to prove inequality (37), we consider

$$
\begin{aligned}
\frac{1+s(z)}{1-s(z)} & =(\lambda+1)\left[\frac{\left(\left(\psi_{\nu, b, c}^{a}(z ; q)\right)_{m}\right)^{\prime}}{\left(\psi_{\nu, b, c}^{a}(z ; q)\right)^{\prime}}-\gamma_{2}\right], \\
& =\frac{1+\sum_{n=1}^{m} \eta_{n} z^{n}-\lambda \sum_{n=m+1}^{\infty} \eta_{n} z^{n}}{1+\sum_{n=1}^{m} \eta_{n} z^{n}} .
\end{aligned}
$$

Therefore,

$$
s(z)=\frac{-(1+\lambda) \sum_{n=m+1}^{\infty} \eta_{n} z^{n}}{2+2 \sum_{n=1}^{m} \eta_{n} z^{n}-(\lambda-1) \sum_{n=m+1}^{\infty} \eta_{n} z^{n}}
$$

and

$$
|s(z)| \leq \frac{(1+\lambda) \sum_{n=m+1}^{\infty}\left|\eta_{n}\right|}{2-2 \sum_{n=1}^{m}\left|\eta_{n}\right|-(\lambda-1) \sum_{n=m+1}^{\infty}\left|\eta_{n}\right|} .
$$

Inequality (37) holds true if $|s(z)| \leq 1$ according to subordination principle. Then, the upcoming inequality,

$$
\sum_{n=1}^{m}\left|\eta_{n}\right|+\lambda \sum_{n=m+1}^{\infty}\left|\eta_{n}\right| \leq 1
$$

implies that $|s(z)| \leq 1$. Since the left-hand side of (52) is bounded above by $\lambda \sum_{n=1}^{\infty}\left|\eta_{n}\right|$; thus, the proof is now completed.

\section{Data Availability}

No data were used in this paper.

\section{Ethical Approval}

This study does not contain any studies with human participants or animals performed by any of the authors.

\section{Conflicts of Interest}

The authors confirm no conflicts of interest.

\section{Authors' Contributions}

The authors contributed to the draft of the manuscript; they have read and approved the final manuscript.

\section{References}

[1] R. M. El-Ashwah and A. H. El-Qadeem, "Some characterizations on the normalized lommel, struve and bessel functions of the first kind," 2017, https://arxiv.org/abs/1712.01689.

[2] A. Baricz, E. Deniz, and N. Yağmur, "Close-to-convexity of normalized dini functions," Mathematische Nachrichten, vol. 289, no. 14-15, pp. 1721-1726, 2016.

[3] E. Deniz and S. G, ren, Geometric properties of generalized Dini functions," Honam Mathematical Journal, vol. 41, no. 1, pp. 101-116, 2019.

[4] A. H. El-Qadeem and M. A. Mamon, "Comprehensive subclasses of multivalent functions with negative coefficients defined by using a q-difference operator," Transactions of A. Razmadze Mathematical Institute, vol. 172, no. 3, pp. 510-526, 2018.

[5] H. Tang, K. Vijaya, G. Murugusundaramoorthy, and S. Sivasubramanian, "Partial sums and inclusion relations for analytic functions involving (p,q)-differential operator," Open Mathematics, vol. 19, no. 1, pp. 329-337, 2021.

[6] R. M. El-Ashwah and A. H. El-Qadeem, "Certain geometric properties of some bessel functions," 2017, https://arxiv.org/ pdf/1712.01687.

[7] B. A. Frasin, "Generalization of partial sums of certain analytic and univalent functions," Applied Mathematics Letters, vol. 21, no. 7, pp. 735-741, 2008.

[8] S. Owa, H. M. Srivastava, and N. Saito, "Partial sums of certain classes of analytic functions," International Journal of Computer Mathematics, vol. 81, no. 10, pp. 1239-1256, 2004.

[9] S. Porwal and K. K. Dixit, "An application of Salagean derivative on partial sums of certain analytic and univalent functions," Acta Universitatis Apulensis, vol. 26, pp. 75-82, 2011.

[10] H. Orhan and N. Yağmur, "Partial sums of generalized Bessel functions," Journal of Mathematical Inequalities, vol. 8, no. 4, pp. 863-877, 2014.

[11] I. Aktas and H. Orhan, "On partial sums of normalized q-bessel functions," Communications of the Korean Mathematical Society, vol. 33, no. 2, pp. 535-547, 2018.

[12] I. Aktas, "Partial sums of hyper-Bessel function with applications," Hacettepe Journal of Mathematics and Statistics, vol. 49, no. 1, pp. 380-388, 2020.

[13] N. Yagmur and H. Orhan, "Partial sums of generalized Struve functions," Miskolc Mathematical Notes, vol. 17, no. 1, pp. 657-670, 2016. 
[14] M. Çağlar and E. Deniz, "Partial sums of the normalized Lommel functions," Mathematical Inequalities and Applications, vol. 18, no. 3, pp. 1189-1199, 2015.

[15] M. U. Din, M. Raza, N. Yagmur, and S. N. Malik, "On partial sums of Wright functions," UPB Scientific Bulletin, vol. 80, no. 2, pp. 79-90, 2018.

[16] M. S. UrRehman, Q. Z. Ahmad, H. M. Srivastava, B. Khan, and N. Khan, "Partial sums of generalized q-Mittag-Leffler functions," AIMS Math, vol. 5, no. 1, pp. 408-420, 2019.

[17] A. H. El-Qadeem and D. A. Mohan, "On some properties of certain subclasses of univalent functions," Italian Journal of Pure and Applied Mathematics, vol. 43, pp. 380-390, 2020.

[18] A. Baricz, "Geometric properties of generalized Bessel functions," Publicationes Mathematicae Debrecen, vol. 73, no. 1-2, pp. 155-178, 2008.

[19] E. Deniz, H. Orhan, and H. M. Srivastava, "Some sufficient conditions for univalence of certain families of integral operators involving generalized Bessel functions," Taiwanese Journal of Mathematics, vol. 15, no. 2, pp. 883-917, 2011.

[20] G. N. Watson, A Treatise on the Theory of Bessel Functions, Cambridge University Press, Cambridge, UK, 2nd edition, 1944.

[21] M. H. Annaby and Z. S. Mansour, Q-Fractional Calculus and Equations, Springer, Berlin, Germany, 2012.

[22] I. Aktas and H. Orhan, "Partial sums of normalized Dini functions," Journal of Classical Analysis, vol. 9, no. 2, pp. 127-135, 2016.

[23] T. Bulboaca, Differential Subordinations and Superordinations, Recent Results, House of Science Book Publication, Cluj-Napoca, Romania, 2005.

[24] P. L. Duren, Univalent Functions, Grundlehen der Mathematischen Wissenschaften, Springer-Verlag, New York, NY, USA, 1983.

[25] S. S. Miller and P. T. Mocanu, Differenatial Subordinations: Theory and Applications, Series on Monographs and Textbooks in Pure and Applied Mathematic, Marcel Dekker, Inc., no. 255, New York, NY, USA, 2000. 